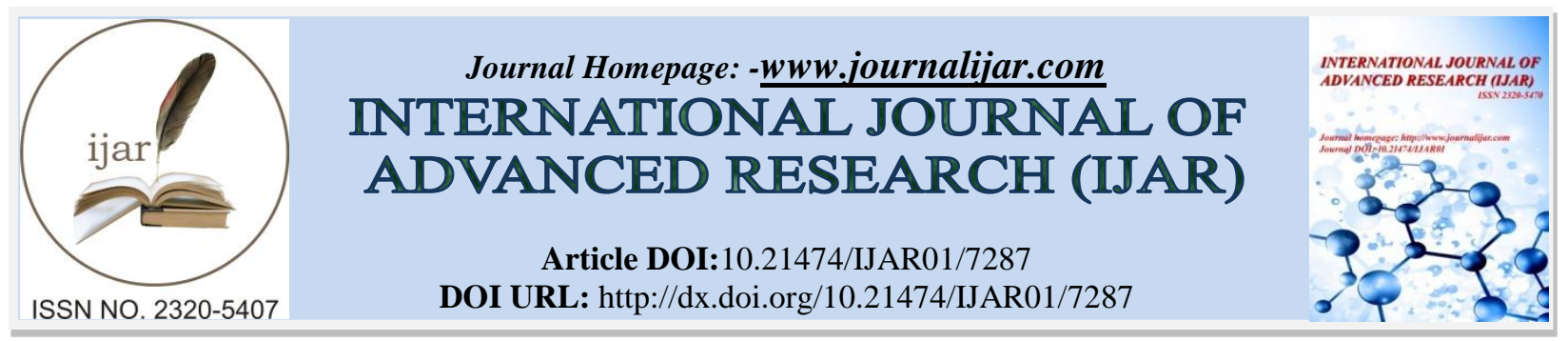

RESEARCH ARTICLE

\title{
MAJOR FINDINGS OF PERIODONTAL DISEASE RELATED TO DIABETES MELLITUS: SYSTEMATIC REVIEW.
}

Júlia Magalhães Pereira ${ }^{1}$, Daniele Barbosa Gonzales ${ }^{1}$, Karina Carradore ${ }^{1}$, Andressa Rocha Codogno, Idiberto José Zotarelli Filho ${ }^{1,2}$, Patrícia Garani Fernandes ${ }^{1,2}$ and Igor Mariotto Beneti ${ }^{1,2}$.

1. University Center North Paulista (Unorp) - São José do Rio Preto - SP, Brazil.

2. Post graduate and continuing education (Unipos), Street Ipiranga, 3460, São José do Rio Preto SP, Brazil 15020-040.

\section{Manuscript Info}

Manuscript History

Received: 14 April 2018

Final Accepted: 16 May 2018

Published: June 2018

Keywords:-

"Periodontal Disease", "Diabetes mellitus", "Diabetes mellitus in periodontitis" and "Clinical Trial".

\section{Abstract}

Background: The prevalence of Diabetes mellitus in the countries of Central and South America was estimated at 27.4 million people and considered for 45 million in 2030. In Brazil, Diabetes mellitus in the population above 18 years increased from $5.3 \%$ to $5.6 \%$ between 2009 and 2016. Objective: was to present the main findings of the relation of periodontal diseases and diabetes mellitus through a systematic review. Discussion: Dental treatment in diabetic patients can be carried out according to their glycemic condition. Almost all patients need to be anesthetized, and diabetic patients are no different, when we use local anesthetics with epinephrine 1: 100,000 are well tolerated. However, epinephrine has pharmacological effect opposite to that of insulin, glucose can therefore increase with use. Must be used with caution epinephrine in hypertensive diabetic patients who have suffered myocardial infarction or who have cardiac arrhythmias. Conclusion: According to literary findings, it is imperative to control Diabetes mellitus, because it is a strong predictor and enhancer of periodontal diseases, and prior to more severe surgical procedures and to apply minimally invasive treatments in these patients.

Copy Right, IJAR, 2018,. All rights reserved.

\section{Introduction:-}

The incidence of Diabetes mellitus (DM) in the countries of Central and South America was estimated at 27.4 million people and considered for 45 million in 2030. In European countries and the United States this will increase, especially in the older age groups due to the increase in life expectancy while in developing countries this increase will occur in all age groups, and the group 45-64 years, the prevalence will be tripled, and doubled in the age groups $20-44$ years and above 65 years $[1,2]$.

In Brazil, data from the risk and protective factors showed that the prevalence of self-reported diabetes in the population above 18 years increased from $5.3 \%$ to $5.6 \%$ between 2006 and 2011 [12]. By analyzing this data according to gender, despite the increase in cases among men who were $4.4 \%$ in 2006 and went to $5.2 \%$ in 2011 , women had a higher proportion of the disease, corresponding to $6 \%$ of this population. In addition, the survey made it clear that the occurrences are more common in people with low education [12].

Corresponding Author:- Júlia Magalhães Pereira.

Address:- University Center North Paulista (Unorp) - São José do Rio Preto - SP, Brazil. 
Moreover, the figures indicate that $7.5 \%$ of people who have up to eight years of study have diabetes, compared with $3.7 \%$ of people over 12 years of study, a difference of $50 \%$ [2-4]. The survey showed also that DM increases with the age of the population: $21.6 \%$ of Brazilians over 65 years reported the disease, a much higher rate than among people aged between 18 and 24 in which only $0.6 \%$ are people with diabetes. It is also estimated that Brazil pass the 8th position, with a prevalence of $4.6 \%$ in 2000 to 6 th position, $11.3 \%$ in 2030. The risk factors related to eating habits and population lifestyle are associated this increase in diabetes globally load $[5,6]$.

In addition, DM has increased in young children and in the last 30 years, mainly Type 1 Diabetes mellitus (T1DM). About 210,000 young people around 20 years of age have diabetes. This represents $0.26 \%$ of the total number of individuals in this age group. About 1 in every 400 to 500 children and adolescents has T1DM [6,7]. The major oral diabetic patient complications include xerostomia, bacterial, viral and fungal infections, delayed wound healing, incidence of caries, gingivitis and periodontal disease, periapical abscess and symptoms of burning mouth [9]. The oral findings in uncontrolled diabetic patients are most likely included with the fluid loss in the urine, altered response to infections, microvascular changes and probably high glucose concentrations in saliva $[10,11]$.

Studies describe increase in incidence and severity of gingivitis and periodontal disease abscess. And there may be minor changes in the blood vessels of the gingival tissues of patients with diabetes. Moreover, adults with diabetes decompensated predisposed periodontal disease offer more severe manifestations than people without diabetes to the same ability [12].

This relationship is not clear in patients with controlled diabetes [13]. Patients with diabetes appear to provide the most severe periodontal disease than those without diabetes, but even so, the differences are not significant. Because of the importance of the dentist to know the clinical manifestations of this disease, diabetes will hold a theme of literature review and its complications in dental treatment $[13,14]$. This on its main forms work aims to discuss dental treatment offered to patients with Diabetes Mellitus highlighting the needs that we have to evaluate increasingly this disease [14].

The objective was to present the main findings of the relation of periodontal diseases and diabetes mellitus through a systematic review.

\section{Methods:-}

Experimental and clinical studies were included (case reports, retrospective, prospective and randomized trials) with qualitative and / or quantitative analysis. Initially, the key words were determined by searching the DeCS tool (Descriptors in Pubmed, Health Sciences, BIREME base) and later verified and validated by MeSh system (Medical Subject Headings, the US National Library of Medicine) in order to achieve consistent search.

\section{Mesh Terms:-}

The words were included "Periodontal Disease", "Diabetes mellitus", "Diabetes mellitus in periodontitis" and "Clinical Trial". The literature search was conducted through online databases: Pubmed, Periodicos.com and Google Scholar. It was stipulated deadline, and the related search covering all available literature on virtual libraries.

\section{Series of Articles And Eligibility:-}

A total of 86 articles were found involving Diabetes mellitus in Dentistry. Initially, it was held the exclusion existing title and duplications in accordance with the interest described this work. After this process, the summaries were evaluated and a new exclusion was held. A total of 42 articles were evaluated in full, and 22 were included and discussed in this study. 


\section{Flow Chart}

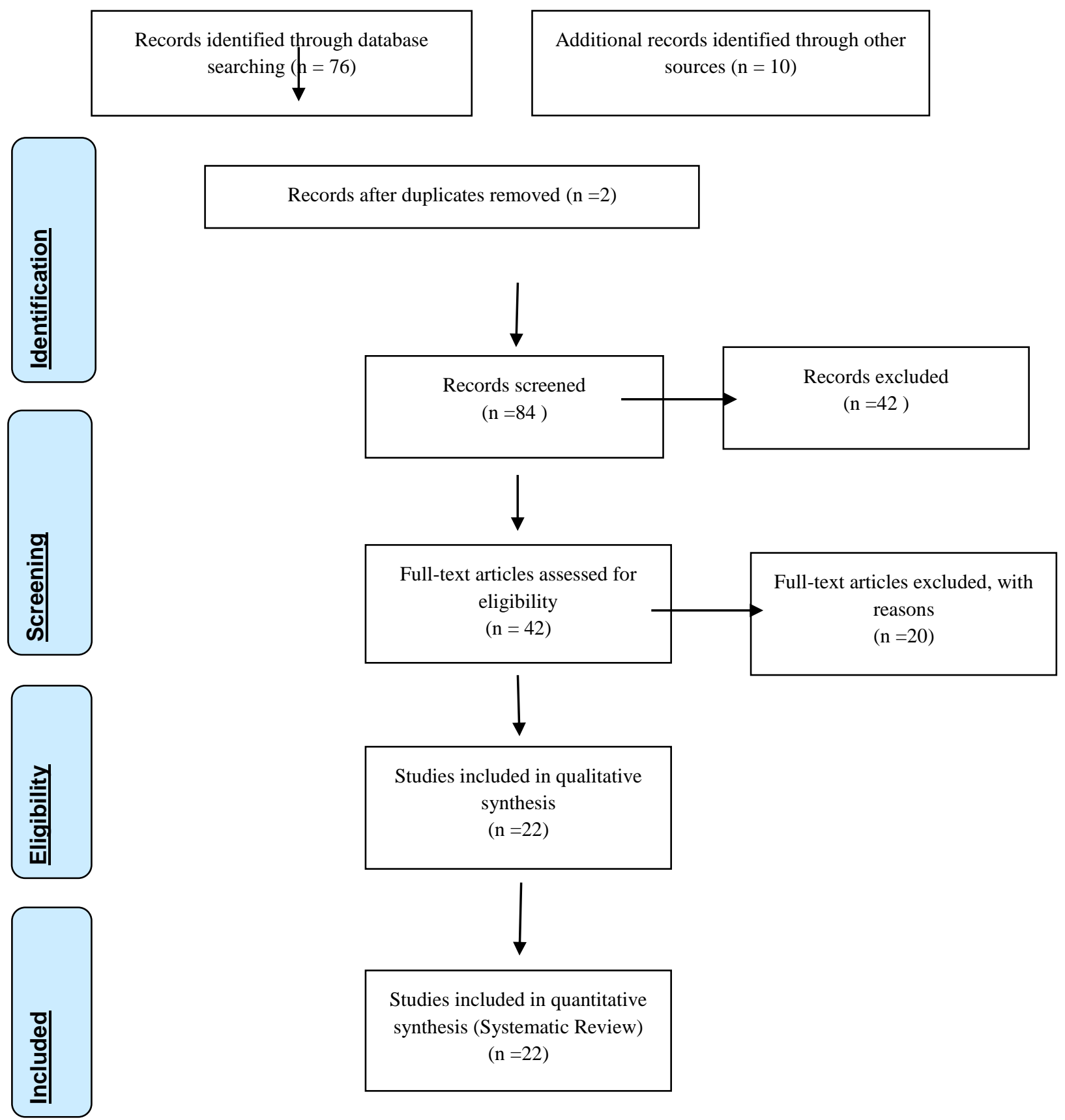

Literature Review:-

A recent study in 2018 showed that the prevalence of severe moderate periodontitis was $36.39 \%$ and $22.71 \%$ among participants with type 2 diabetes and without diabetes, respectively. type 2 diabetes was significantly associated with severe moderate periodontitis or (or $=1.47$; ic 95\%: 1.18-1.82) among knowledge even after adjusting for demographics, socioeconomic status, and oral health behaviors [1]. In addition, different relationships of moderate and severe periodontitis with body mass index and toothbrush use were found between genders. Therefore, current findings suggest that important improvements in the development of gender-specific prevention strategies such as home care, reducing the high prevalence of periodontal disease and maintaining good oral health are vital and are 
especially important for male and female diabetic patients. at high risk of developing diabetes, such as those who are obese [1].

In addition, another study showed that there was a statistically significant change in all parameters observed clinically in both groups. Glycemic status also showed a statistically significant reduction for fasting blood sugar (P $<0.001)$ in the intragroup comparison [2]. The intragroup comparison showed a statistically significant reduction (P $<0.001)$ for serum lipids, whereas intergroup comparison showed a statistically significant reduction after treatment only at CT and LDL levels ( $\mathrm{P}<0.02$ and $<0.012$ ). Therefore, this study shows that fenugreek powder can be used as adjuvant to SRP to control the glycemic state and serum lipid levels in patients with uncontrolled DM [2].

Further, the result was clinically evaluated, the need for periodontal care that affect daily life. The relationship between outcome and adjusted risk factors for personal history and disability was assessed using ordinal regression. Healthy and disabled people had a high need for periodontal care, with $66.8 \%$ [19]. Current smokers were more likely and health insured persons were less likely to need periodontal care that would affect daily life regardless of whether the disability was considered. Most adults needed periodontal care, and people with disabilities experienced a greater impact on life [19].

This disease is classified by hyperglycemia and complications include renal and ocular microvascular disease and various clinical neuropathies [1]. The DM is linked to premature chronic macrovascular disease and severe microvascular disease [1,2]. The metabolic component involves rise in blood glucose associated with changes in lipoprotein metabolism as a result of absolute or relative insulin deficiency. Maintaining good glycemic control can prevent or delay the development of microvascular complications of diabetes [2-5].

Dryness and irritation of the oral mucosa, reduction of salivary flow and flora changes, with predominance of Candida albicans, are the most evident oral findings in the diabetic patient. Thus, Diabetes mellitus is one of the systemic conditions aggravating the periodontal response to bacterial plaque. In addition, it was observed that patients with improvement in the gingival bleeding index presented a decrease in glycosylated hemoglobin levels [19].

In addition, DM is a disease that presents metabolic and vascular components [6,7]. Retinopathy and nephropathy comprise any complications that arise in the course of time in virtually all diabetics. These complications result in severe morbidity and are so peculiar diabetes that their presence is used to classify the type of diabetes [7,8]. The DM is a disease of broad importance to dentists because they are health professionals able to detect new cases of the disease [9].

In this context, dentists are also able to provide dental treatment for patients with diabetic sensibly without putting them at risk or causing damage to the control of diabetes [10]. An essential look to be identified in diabetic dental patients is the severity and the level of glycemic control, as well as the presence of complications coming from the disease, to treat them properly [13]. Knowing the level of the patient's blood glucose at the time of completion of the dental treatment is paramount. In addition, the diagnosis of DM is acquired through fasting glucose. Patients who have very close glucose levels of the upper limit of normal range are subjected to glucose tolerance test [14].

Also the DM therapy is to support the interrelation of three measures: diet, exercise and targeted use of drugs, depending on the type of diabetes and other factors. The most recommended drugs are oral hypoglycemic agents, including sulfonylureas, first choice for type 2 diabetics not obese [15]. Metformin is an oral antidiabetic preferred to treat type 2 diabetics, obese or overweight $[2,16]$.

Moreover, the insulin used most commonly in T1DM there are several types of preparations can be natural or obtained by bacterial synthesis (human insulin). The duration of insulin may be rapid, slow or ultra-slow-acting, being uniquely prescribed by physicians. The dentist should never change the dosage of hypoglycemic agents, especially insulin, even in emergency situations, can induce hypoglycemia [17].

In both types of diabetes mellitus type I and II metabolism of all nutrients is changed. The basic effect of the absence of insulin or insulin resistance on glucose metabolism and prevent the efficient uptake and use of glucose by most cells of the body except the brain. As a result, the blood glucose concentration increases, cell glucose utilization drops even more and the use of lipids and proteins increases [17,18]. 
Thus, the main symptoms of diabetes are: The polyuria, polydipsia, the polyphagia, weight loss are insulin deficiency results. Insulin acts necessarily in regulating the metabolism of carbohydrates, fats and proteins. Insulin deficiency results in decreased blood glucose entry into the tissues and an increased blood glucose level $[5,6]$.

Besides the increase in blood glucose levels leads to an increase of glucose intended kidneys. The lack of resorption of all this exaggeration of glucose by the kidney resulting in glucosuria, triggering osmotic diuresis and an increase in urine output, to be compensated by an increase in fluid intake [6]. The continuous loss of glucose in the urine also results in weight loss despite increased food intake. In the abnormality in utilization of glucose, insulin deficiency also causes abnormality in the metabolism of proteins [7].

Also the clinical signs of diabetes include xerostomia, bacterial, viral and fungal infections, delayed wound healing, incidence and severity of dental caries, gingivitis and periodontal disease, periapical dental abscesses and burning. In addition to the diseases that are related to diabetes such as atherosclerosis, increased susceptibility to infection, diabetic retinopathy, cataracts, hypertension and chronic kidney disease, are deeply associated with lipid levels and blood glucose, some doctors also use lowering drugs lipid to help avoid these changes [2,3].

\section{Discussion:-}

Dental treatment in diabetic patients can be carried out according to their glycemic condition. Almost all patients need to be anesthetized, and diabetic patients are no different, when we use local anesthetics with epinephrine 1: 100,000 are well tolerated. However, epinephrine has pharmacological effect opposite to that of insulin, glucose can therefore increase with use. Must be used with caution epinephrine in hypertensive diabetic patients who have suffered myocardial infarction or who have cardiac arrhythmias [3].

For diabetic patients well-controlled surgical antibiotic prophylaxis is not routinely, by adopting a sterilization protocol or local antisepsis. Prophylactic use of antibiotics in diabetic should only be considered in patients with decompensated disease with blood ketoacidosis and ketonuria, when the functions of neutrophils are decreased. You cannot generalize such conduct to all diabetics, each case must be analyzed carefully $[5,6]$

Further, acute complications in diabetes is the most important insulin shock, characterized by an acute hypoglycemia, which can be life-threatening, because it develops very quickly, resulting in loss of consciousness and possibly seizures. With the decrease in blood glucose with blood levels $<40-50 \mathrm{mg} \mathrm{dL}-1$ seems to be the crucial factor in the increase in clinical symptoms of hypoglycemia, excluding the central nervous system of its fundamental energy source [8-10].

It is important to identify the signs and symptoms of hypoglycemia in diabetic patients is essential that the dentist knows differentiates them from those associated with the state of ketoacidosis, an emergency situation of rare occurrence in the office, it drags days or weeks to develop, not less important than insulin shock [10].

\section{Conclusion:-}

According to literary findings, it is imperative to control Diabetes mellitus, because it is a strong predictor and enhancer of periodontal diseases, and prior to more severe surgical procedures and to apply minimally invasive treatments in these patients.

\section{Competing Interests:-}

The authors none declare. 


\section{References:-}

1. Liu Y, Yu Y, Nickel JC, Iwasaki LR, Duan P, Simmer-Beck M, Brown L. Gender differences in the association of periodontitis and type 2 diabetes. Int Dent J. 2018 May 22. doi: 10.1111/idj.12399.

2. Sundaram G, Ramakrishnan T, Parthasarathy H, Raja M, Raj S. Fenugreek, diabetes, and periodontal disease: A cross-link of sorts! J Indian Soc Periodontol. 2018 Mar-Apr;22(2):122-126. doi: 10.4103/jisp.jisp_322_17.

3. Lee JY, Giannobile WV. Taxes on Sugar-Sweetened Beverages: A Strategy to Reduce Epidemics of Diabetes, Obesity, and Dental Caries? J Dent Res. 2016; 26.

4. Mishra V, Shettar L, Bajaj M, Math AS, Thakur SL. Interlinking Periodontitis and Type 2 Diabetes Mellitus by Assessment of Crevicular Visfatin Levels in Health and in Disease Before and After Initial Periodontal Therapy. J Clin Diagn Res. 2016; 10(8):ZC67-71.

5. Alexander RE. Routineprophylacticantibiotic use in diabetic dental patients.JCalifDent Assoc; 27(8):611-8, 1999.

6. Andrade ED; Ranali. J, organizações. Emergências médicas em odontologia. 3 edição;São Paulo: Artes Médicas;66-8, 2011.

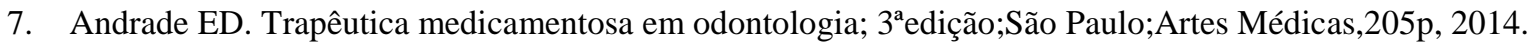

8. Bloomgarden ZT. Clinical diabetic neuropathy. Diabetes Care; 28:2968-2974p, 2005.

9. Fiske J. Diabetes mellitus and oral care. DentUpdat ;31:190-196-198p, 2004.

10. Guyton AC. Tratado de fisiologia médica; 9aedição;Rio de Janeiro; Guanabara Koogan,892p, 2011.

11. Guyton AC. Tratado de fisiologia médica; 10ª edição;Rio de Janeiro;Guanabara Koogan,999p, 2012.

12. Lemmark A. Type 1 diabetes-Does suppressing T cells increase insulin? N Engl J Med,29:161-167p,2005.

13. Little JW; Falace, D. A.; Miller; C. S.; Rhodus; N. L. Manejo odontológico do paciente clinicamente comprometido; 7ªedição;Rio de Janeiro; Mosby Elsevier,205p,2009.

14. Milley DD, Terezhalmy GT. The patient with diabetes mellitus: Etiology, epidemiology, principles of medical management, oral disease burden, and principles of dental management. Quintessence Int; 36:779-795p,2005.

15. Ministério Da Saúde. Secretaria de Atenção à Saúde. Departamento de Atenção Básica. Diabetes Mellitus.Caderno de Atenção Básica \{n.36\} Brasília(DF):Secretaria de Atenção à Saúde;2013.

16. Nathan DM, Cleary PA, Backlund JY, et al. Intensive diabetes treatment and cardiovascular disease in patients with type 1 diabetes. N Engl J Med;353:2643-2653p,2005.

17. Nogid, A. Pham DQ. Adjunctive therapy with pramlintide in patients with type 1 or type 2 diabetes mellitus. Pharmacotherapy; 26 (11):1626-1640p,2006.

18. Ramsier, CA. Potential impact of subject-based risk factor control on periodontitis . JClinPeriodontol;32 (suppl 6):283-290p,2005.

19. Sonis, S.T.; Fazio, R. C.; Fang, L. Princípios e prática de medicina oral. 2ªedição; Rio de Janeiro: Guanabara Kooga, 491p,1996.

20. El Tantawi M, AlAgl A. Disability and the impact of need for periodontal care on quality of life: A crosssectional study. J Int Med Res. 2017 Jan 1:300060517715376. doi: 10.1177/0300060517715376.

21. Yost S, Duran-Pinedo AE, Krishnan K, Frias-Lopez J. Potassium is a key signal in host-microbiome dysbiosis in periodontitis. PLoS Pathog. 2017 Jun 20;13(6):e1006457. doi: 10.1371/journal.ppat.1006457.

22. Salman S, Khan K, Salman F, Hameed M. Effect Of Non- Surgical Periodontal Treatment On Glycemic Control Among Type 2 Diabetes Mellitus Patients With Periodontitis. J Ayub Med Coll Abbottabad. 2016 OctDec;28(4):442-445. 